\section{XAVIER ZUBIRI E IL PROBLEMA DELLA VERITÀ 'ATTUALE'}

\author{
Paolo Ponzio \\ Università degli Studi di Bari Aldo Moro \\ paolo.ponzio@uniba.it
}

Cómo citar este artículo/Citation: Ponzio, P. (2016). Xavier Zubiri e il problema della verità 'attuale'. Arbor, 192 (780): a334. doi: http://dx.doi.org/10.3989/arbor.2016.780n4010

Recibido: 25 julio 2014. Aceptado: 01 septiembre 2015.

RESUMEN: El presente artículo quiere indagar el problema de la verdad en el pensamiento de Xavier Zubiri, desde sus inicios. Por esta razón, incluso si el objetivo es explorar el concepto de verdad en su última declinación, es decir, dentro de la trilogía de la inteligencia sentiente (1980-1983), no se puede ignorar los ensayos anteriores del filósofo, para evaluar plenamente la originalidad de su pensamiento con respecto a la filosofía del siglo XX.

PALABRAS CLAVE: Zubiri; verdad; actualidad; inteligencia sentiente.

\section{XAVIER ZUBIRI AND THE PROBLEM OF 'ACTUAL' TRUTH}

Copyright: (c) 2016 CSIC. Este es un artículo de acceso abierto distribuido bajo los términos de la licencia Creative Commons Attribution (CC BY) España 3.0.
ABSTRACT: This article aims to investigate the problem of truth in the thinking of Xavier Zubiri, from its beginnings. For this reason, even if the goal is to explore the concept of truth in his last declination, i.e., in the trilogy of sentient intelligence (19801983), previous essays by the philosopher must not be ignored when assessing his originality with respect to Twentieth century philosophy.

KEYWORDS: Zubiri; truth; actuality; sentient intelligence. 
"Quando Pilato domandò a Cristo: “Che cos'è la verità?", formulò la domanda che ha determinato la sorte del cristianesimo in Europa. Per questo il cristianesimo si è esteso intellettualmente in Europa: perché ha potuto dare un significato alla parola "verità" e ha creduto nella possibilità di accogliere al suo interno la aletheia dei greci» (Zubiri, 2002, p. 57).

Il brano con cui abbiamo voluto introdurre il problema della verità nel pensiero di Xavier Zubiri, tratto dal lungo saggio intitolato Sobre el problema de la filosofia, delinea in modo chiaro quale sia l'orizzonte problematico nel quale si pone la questione della verità all'interno degli scritti del filosofo spagnolo, sin dai suoi esordi. La domanda di Pilato sembra essere il punto emblematico di contatto tra filosofia greca e cristianesimo. II concetto di verità, di aletheia, viene ora interpretato alla luce del cristianesimo. Cosa ha significato questo - si domanda Zubiri - all'interno della storia della filosofia occidentale?

\section{L'ORIZZONTE DELLA FILOSOFIA OCCIDENTALE: LA VERITÀ IN QUANTO MUTABILITÀ E “NIHILIDAD”}

La visione prospettica dell'uomo greco, entro cui si è mosso l'orizzonte della filosofia, è la mutabilità del reale. Ogni cosa è mutevole e anche l'uomo si coglie allo stesso modo. Sembrerebbe, così, che vi sia un solo aspetto che differenzi l'uomo dalle altre cose: la sua capacità di parlare, di esprimere le cose e il suo essere tra esse. Il logos dell'uomo si caratterizza, dunque, nel poter manifestare l'essere delle cose così come si presenta realmente: I'aletheia greca, la verità delle cose, è allora questo esser-patente che ha nella mutabilità, nel movimento, il suo "per sempre", la sua totalità. Tutta la visione dell'uomo greco è sempre visione di una totalità, di un orizzonte in cui "l'uomo vede le cose quando le esprime» (Zubiri, 2002, p. 44), ed è proprio la visione diretta di qualcosa che è per sempre, ciò che Parmenide chiamò noein. Ciò che rende possibile all'uomo esprimere le cose, ciò che rende possibile il logos, è questo muoversi nella visione di ciò che è per sempre. Nel vedere le cose in ciò che sempre sono, l'uomo scopre le cose stesse nella loro verità, e ciò che in verità sono è ciò che è visto nella visione: l'essere e la visione di ciò che è sempre, sono inseparabilmente unite. Di qui la domanda filosofica distintiva di tutta la filosofia greca: in cosa consiste ogni realtà in quanto tale, cioè, in quanto è sempre? O più sinteticamente: cos'è ciò che è. In questo domandare vi sono riassunti, anzi unificati, i due temi essenziali del pensiero ellenistico: il tema del reale nella sua singolarità, nel suo essere individuale, e il tema della totalità. E tale unificazione non può che palesarsi nel noein parmenideo che ha nel pensiero aristotelico il suo punto teoretico più elevato. Tutta la metafisica di Aristotele, infatti, non è solo scienza delle cose, ma scienza dell'essere, vale a dire, scienza delle cose in quanto sono: "Guardando le cose perse nella mutabilità del tutto, nell'orizzonte totale di ciò che sempre è, il greco si domandò: ti to on» (Zubiri, 2002, p. 46).

Il filosofo greco, dunque, è colui che vive in questa visione del "sempre è" delle cose, e la stessa filosofia consiste nel mantenersi in questa visione, che è, ultimamente, visione del divino. È questo il culmine del pensiero pre-cristiano: nel noein si costituisce, a un tempo, l'essere sempre delle cose in quanto tali e la radice della sufficienza umana, cioè, della sua libertà ${ }^{1}$.

L'orizzonte della filosofia dopo l'avvenimento del cristianesimo è completamente differente. Al concetto di movimento, di variabilità, si sostituisce quello di nihilidad. A tema non c'è più qualcosa (Ón) che muta ma il niente che desidera essere, e dunque, la domanda filosofica non si porrà innanzitutto nel determinare ciò che cambia o ciò che si muove, ma nel chiedersi il perché dell'essere: "Per il greco, il mondo è qualcosa che varierebbe; per l'uomo della nostra Era è un niente che pretende di essere» (Zubiri, 2002, p. 49). Si potrebbe, così, dire che mentre il pensiero greco inizia a filosofare a partire dall'essere, quello occidentale cristiano inizia a filosofare a partire dal niente. Diviene allora imprescindibile dover giustificare come le cose giungono all'essere: non più il problema di che cosa sia ciò che è, ma il problema dell'essere in quanto tale.

«Quando il greco si domanda cos'è il mondo, parte dalla supposizione che mondo sta lì, e ciò di cui si tratta è giustamente di verificare che cos'è. Quando un uomo della nostra Era, invece, si domanda cos'è il mondo, la prima cosa che pensa è, effettivamente, che esso sia stato, che avrebbe potuto non esser stato, né essere ciò che è, né come è» (Zubiri, 2002, p. 50).

A partire, pertanto, dall'avvenimento del cristianesimo l'uomo assume un modo di esistere completamente nuovo, tanto che la domanda filosofica si pone all'interno di un orizzonte inimmaginabile all'uomo greco. È vero che sin dai primi secoli si è parlato di una certa commistione del pensiero cristiano con quello greco, ma occorrerà domandarsi il motivo di quest'apertura alla filosofia e quali sono state le conseguenze per la stessa filosofia.

Di certo il pensiero cristiano trae la sua origine non solo dalla filosofia greca, ma anche da una tradizione a essa parallela, quella giudaica, che si presenta del 
tutto differente, se non opposta, - come mentalità e orizzonte esistenziale -, rispetto a quella ellenistica. Innanzitutto, la posizione dell'ebreo nel mondo: egli vive prossimo tra i prossimi, l'altro non è qualcosa di a sé stante, un momento della natura, come per il greco, bensì si situa in rapporto con me. Non il prossimo in quanto tale, ma il mio prossimo: un prossimo cui appoggiarsi, il prossimo della mia tribù che dà sicurezza, stabilità alla mia vita. Questa fiducia, questa sicurezza è alla base del concetto di verità: "La verità si presenta così all'ebreo come fedeltà, compimento di una promessa, veracità» (Zubiri, 2002, p. 53). In questo modo, la verità più che essere qualcosa che si vede, è qualcosa che avviene, qualcosa che accade, cosicché l'organo della verità non è più il logos ma la fiducia in un avvenimento futuro. È questo il senso della storia che pervade l'intero percorso del popolo ebraico: non un movimento tra le cose, ma la certezza di una storia che mostrerà il vero volto delle cose, della realtà, che non potrà più essere considerata nella sua natura di eidos, essenza, ma in quanto destino. Il tempo della storia per l'ebreo non è più il tempo del semplice movimento delle cose, il tempo tra le cose, bensì il tempo del destino, verso il destino. Un destino che non è sinonimo di fatalità, di casualità; è, invece, una promessa, una "parola" che viene incontro all'uomo. A differenza dell'idea di tempo come movimento, che implica da sé l'essere momento della natura, il tempo inteso in quanto destino conduce a colui da cui dipende l'essere stesso delle cose, il signore di tutto. Questi, tra l'altro, non solo è un Dio che esiste - contro un Dio irreale - ma è anche un Dio che promette, un Dio che compie la sua promessa nella storia del popolo, e colui che penetra il suo volere non può più essere definito saggio, sapiente, bensì profeta poiché trasmette ciò che ha udito, annunciando e pre-annunciando al popolo la parola divina.

Fedeltà e disvelamento, l'amen ebraico e l'aletheia greca trovano il loro fondamento e, insieme, la loro riconciliazione nell'incontro di Cristo con Pilato, nel primo contatto che il mondo greco-romano ha con l'avvenimento cristiano nel suo farsi storico. Due concezioni parallele trovano il loro punto di incontro in questa conversione, in questa lenta ma progressiva trasformazione del significato del concetto di verità nella cultura occidentale. Quale sarà il cammino lento e tortuoso di tale trasformazione lo chiarirà la storia nel corso dei secoli.

Emerge qui, tuttavia, un dato originario che quasi sempre viene sottinteso o, ancor peggio, coscientemente taciuto: la conoscenza della verità è possibile solo mediante qualcosa di precedente alla stessa conoscenza, mediante un incontro che precede il conoscere. Conosciamo la verità poiché è la verità stessa che si fa incontro all'uomo, cosicché la conoscenza della realtà è, innanzitutto, conoscenza del suo rapporto con Dio, del suo esser dato, creato. L'idea greca della conoscenza in quanto teoria cede, pertanto, il posto alla contemplatio, tanto che la domanda originaria del nuovo sapere filosofico è la seguente: come è possibile che le cose siano qualcosa e contemporaneamente niente? Come è possibile che sorgano all'essere dal niente? In questo modo la questione dell'essere delle cose non ha più a che fare con il problema della traiettoria attuale del movimento, ma con l'avverarsi della creazione: "Essere è esser-creato» (Zubiri, 2002, p. 121). II "quid est ens" della metafisica greca si trasformerà nel "quid est esse qua creatum esse" e pervaderà tutta la storia della metafisica post-ellenica.

La traiettoria dell'orizzonte filosofico europeo da Agostino a Hegel - sostiene Zubiri nelle ultime pagine del saggio Sobre el problema de la filosofía - non può fare a meno di questa ulteriorità. L'uomo non può incontrarsi in modo immediato con se stesso, ma mediante il riferimento a Dio, cioè con lo sguardo fisso sull'infinito.

«La filosofia da Descartes a Hegel è una dialettica dello spirito finito che vuole conquistare intellettualmente l'infinitudine, messa in crisi dal distanziarsi di Dio. Questa distanza di Dio ha portato inesorabilmente a una divinizzazione dell'uomo. Per questo ancora Hegel ha potuto dire che la filosofia non è se non l'esposizione dello spirito di Dio prima della creazione. È possibile questo?, o se si vuole, non è possibile l'esistenza di una filosofia pura che non sia se non una pura filosofia?» (Zubiri, 2002, p. 124).

Ecco, dunque, la questione fondamentale. La parabola dell'orizzonte filosofico europeo moderno con il pensiero hegeliano giunge alla sua inesorabile destinazione: I'infinitizzazione del finito. Di qui la domanda finale con cui si chiude Sobre el problema de la filosofia: è possibile l'esistenza di un nuovo orizzonte che non sia quello hegeliano di una pura filosofia?

È questa la domanda che pervade l'iter filosofico zubiriano, e a cui il filosofo spagnolo darà una risposta iniziale attraverso l'incontro con la fenomenologia husserliana che diviene il primo termine di confronto diretto. Ma anche qui occorrerà preliminarmente porsi la domanda: cosa si intende per fenomenologia? "La fenomenologia - scrive il filosofo spagnolo - inizia dal presupposto che la filosofia è innanzitutto un 
modo speciale di conoscere le cose» (Zubiri, 2002, p. 124). Così Zubiri definisce da subito la fenomenologia, intendendola in questo "modo speciale" una condizione completamente differente rispetto a tutta la storia metafisica classica e all'epistemologia moderna. Per poter situare tale nuovo modo di conoscenza delle cose, occorrerà allora sintetizzare le diverse ma decisive concezioni del "sapere umano" così come si sono mostrate nel corso della storia del pensiero. Non sembri una semplice ricostruzione storiografica; ciò che Zubiri vuole mostrare è l'ineluttabile necessità del problema gnoseologico all'interno di ogni filosofare. Il chiedersi che cos'è il sapere è il problema della filosofia, non uno dei problemi tra altri. Ciò porterà, tra l'altro, il filosofo spagnolo a ripresentare quest'ultima parte del saggio all'interno di Naturaleza, historia, Dios, intitolandolo ¿Qué es saber?².

Nella storia del pensiero occidentale vi sono, secondo Zubiri, tre diversi modi di significare il sapere: in quanto discernere, in quanto definire e in quanto intendere. E tutti questi modi dipendono da altrettante forme del filosofare: per la filosofia di Parmenide il sapere ha significato discernere ciò che è da ciò che non è o, come egli stesso diceva, l'essere dal sembrare d'essere. Per Platone, invece, il discernere non sembrava più sufficiente, poiché non solo discerniamo una cosa dalla sua apparenza, ciò che è da ciò che non è, ma circoscriviamo i limiti e i confini, la definiamo. Sapere, dunque, non come discernere ma come definire. Infine, per la filosofia aristotelica anche l'idea del sapere come definizione aveva bisogno di essere nuovamente ricompresa: il sapere è sempre qualcosa in più rispetto al discernere e al definire, poiché sappiamo qualcosa in modo completo non solo quando sappiamo il "che cosa" è, ma soprattutto quando ne conosciamo anche il "perché" è. Non un semplice "che cosa", ma un "che cosa" che è tale "perché" le cose "sono" così e non in un altro modo. In tal modo, la cosa non l'abbiamo solo distinta e definita, l'abbiamo anche intesa, non solo l'abbiamo mostrata, bensì l'abbiamo anche di-mostrata. Questo sapere inteso come di-mostrazione, a sua volta, ha tre momenti: il raziocinare (raciocinar), cioè l'impiego rigoroso della logica come organon dell'intero sapere reale; l'attività della mens, o nous, volta a far vedere la cosa così come essa è, cioè a partire dai suoi principi; e, infine, l'impressione reale delle cose, perché non abbiamo solo bisogno di compiere un discorso sulle cose o di di-mostrarne il sapere attraverso principi, occorre conoscere come ciò che veramente è, sia qui e ora quella cosa e non un'altra: "Occorre intendere non solo "ciò che è" la cosa, ma anche "la cosa che è"; non solo l'essenza, ma la cosa stessa» (Zubiri, 2002, p. 199). Ora il principio delle cose è questo "essere veramente": è, cioè, il tutto. Ciò che si chiama, allora, in modo determinato "ciascuna cosa" è ciò in cui il principio, il tutto si è concentrato. In tal modo, in ogni cosa, in principio, c'è già tutto; ogni cosa non è altro che uno specchio che riflette, quando la luce della mente lo colpisce, il tutto. Questi è, dunque, nella cosa specularmente, per cui sapere una cosa dal punto di vista dei suoi principi, cioè dal punto di vista del tutto, è saperla speculativamente. Insomma, intesa la cosa, co-intendiamo il tutto. Questa modalità di co-intendere è ciò che si chiama "sistema": sapere qualcosa è saperlo sistematicamente, nella sua co-intesa con il tutto. È stato questo il genio dell'idealismo tedesco, che ha avuto il merito di chiudere questa dinamica aperta dalla mens aristotelica. Quest'ultima non solo ha scoperto i principi di ciò che vede, ma il principio stesso della visibilità; la mente vede se stessa riflessa nello specchio delle cose in quanto sono: «Nelle cose che sono veramente, la verità si rende patente [se patentiza] in modo puro» (Zubiri, 2002, p. 200). II sapere è così uno scoprirsi della mente a se stessa. Fin qui la parabola che da Aristotele a Hegel ha, secondo Zubiri, portato alle estreme conseguenze il rifiuto di ogni conoscenza che abbia a che fare con la sensibilità:

«La prima metà del XIX secolo è consistita - scrive in Filosofía y metafísica - nel furore romantico di questa speculazione. Lo scienziato fu l'elaboratore di sistemi speculativi. Di contro a lui si alzò la voce del "ritorno alle cose" [vuelta a las cosas]. Sapere non è raziocinare né speculare: sapere è attenersi modestamente alla realtà delle cose» (Zubiri, 2002, p. 200).

Ecco allora la questione: la cosa stessa, vale a dire, la cosa intesa nella sua realtà. Tutto il sapere speculativo, dai primi abbozzi parmenidei sino alla grande sintesi idealistica, ha svolto il problema gnoseologico a partire dalla verità senza riuscire ad arrivare alle cose. Ci si è arrestati alle idee, credendo che conoscere l'idea di una cosa equivalesse a conoscere la cosa in se stessa. In tal modo emerge il grande compito che si assume il sapere fenomenologico: «non solo la "verità della realtà", ma anche la "realtà della verità". Occorre che le cose siano comprese "nella realtà di verità"» (Zubiri, 2002, p. 201).

Se queste sono le premesse filosofiche da cui occcorreva partire in questa nostra ricognizione del problema della verità mi sembra interessante annotare come tale compito fenomenologico sia stato raccolto da Zubiri e trasformato all'interno della trilogia sull'Inteligencia sentiente. 


\section{DALLA VERITÀ REALE, ALL'INCONTRO CON LA VERITÀ RAZIONALE}

«La cosa reale è appresa come reale in e per se stessa: è "da sé" ciò che è. Dato che questo momento di formalità è un prius delle cose, risulta che la realtà non consiste formalmente né si esaurisce inevitabilmente nell'essere intelligita. Perciò, per intelligire ciò che la cosa realmente è, diremo che l'intellezione è vera. Ciò che la mera attualizzazione del reale aggiunge alla realtà è, dunque, la sua verità» (Zubiri, 2008, pp. 327-329).

Il tema della verità in Zubiri - così come emerge nel presente brano di Inteligencia y realidad - è una stretta conseguenza dell'attualità intellettiva. Vi è una relazione diretta tra attualità e verità; anzi la verità sembra essere quasi una "produzione" dell'attualità all'interno della realtà. In questo modo, il problema della verità non viene ad aggiungersi all'atto intellettivo, non è un altro componente in più, bensì si configura come una speciale visione dello stesso atto intellettivo con il quale si è appreso primordialmente il reale.

Ma qual è il significato che Zubiri dà a questo termine? Se rispetto all'atto intellettivo si è cercato di giungere a determinazioni originarie tali da ricondurre ogni discorso a questioni di fondamento, per lo più omesse dall'orizzonte filosofico moderno, allora anche il significato di verità non potrà che emergere in un approfondimento di quella che è la realtà "da sé". Normalmente si considera il tema della verità connesso alle qualità di un'affermazione, dimenticando che l'affermazione, in quanto tale, è soltanto un modo di intellezione. II concetto di verità, invece, non concerne solo il modo affermativo di intellezione, ma tutta l'intellezione in quanto tale. La verità, allora, è l'intellezione in quanto apprende il reale presente come reale. Essa, in quanto tale, non aggiunge nulla alla realtà se non la mera attualizzazione. Con ciò Zubiri non vuol dire che verità e realtà sono la stessa cosa. Non si tratta né di realtà identiche, né di realtà correlate, poiché se è vero che ogni verità implica la realtà, non ogni realtà implica la verità. È la realtà, quindi, il fondamento della verità, ciò che dà verità all'intellezione. "Verità è puramente e semplicemente il momento della reale presenza intellettiva della realtà » (Zubiri, 2008, p. 329): vale a dire, è un momento di mera attualizzazione del reale. Questa verità che Zubiri chiama verità reale, in opposizione sia alla concezione kantiana - secondo cui la verità è solo coscienza oggettiva - sia a quella di matrice tanto razionalista quanto idealista - secondo cui la verità consisterebbe nell'affermazione logica, dipende strettamente dall'apprensione primordiale di realtà, e rappresenta uno dei maggiori punti di novità della filosofia di Zubiri, determinando anche l'intero impianto concettuale del problema della verità negli altri due modi di intellezione senziente: il logos e la ragione. Ma come si caratterizza tale verità reale? In quanto verità primaria e radicale dell'intelligenza senziente tale verità reale, pur non aggiungendo nulla alla realtà in quanto tale, non si identifica con essa. È una specie di qualificazione intrinseca che trova il suo impianto nell'attualità. Verità è, allora, il fatto che le cose rimangono presenti nell'intellezione come reali. Verità è, così, ultimamente ratifica: "Ecco la natura essenziale della verità reale: il reale sta "ne" l'intellezione, e questo "in" è ratifica» (Zubiri, 2008, p. 333).

Tale concetto di ratifica, secondo Zubiri, è di estrema rilevanza poiché approfondisce la distanza tra un'idea di verità in quanto riferita a quanto di una cosa reale si concepisce o si afferma, e l'idea di una verità reale originaria, tale solo in quanto attualizza il reale in quanto tale. È questa l'idea di una verità semplice, una verità con la quale non si fuoriesce dal reale verso un concetto, verso un'affermazione o verso una ragione ${ }^{3}$.

La verità reale partecipa della tripartizione della realtà distinguendosi anch'essa in tre dimensioni - la ricchezza, il che cosa e la stabilità - da cui dipendono i modi attraverso cui può dirsi la verità stessa. La totalità della realtà, infatti, si ratifica in ricchezza secondo una modalità specifica che il filosofo spagnolo denomina manifestazione; mentre il che cosa dipende dalla ratificazione della coerenza della realtà secondo una modalità denominata fermezza. Infine la realtà durevole si ratifica nella stabilità secondo una modalità propria, chiamata constatazione. Questi tre modi propri di ratifica - manifestazione, fermezza e constatazione - derivano poi, spiega Zubiri, direttamente dall'uso linguistico differente che la stessa idea di verità ha assunto nelle più importanti lingue antiche, il greco, il latino e l'ebraico. E qui, come si vede, ritorna l'idea di una corrispondenza tra le varie tradizioni culturali e filosofiche. La radice del termine verità costituisce, infatti, un'importante test per verificare l'attendibilità delle tre modalità con le quali la realtà viene ratificata: l'essere greco (es-), la certezza latina (uer-) e l'esser patente ebraico (la-dh-) rappresentano dal punto di vista linguistico quell'unica idea di verità reale cui allude il filosofo. Ogni verità reale, infatti, possiede indissolubilmente le tre dimensioni che sono congeneri come momenti strutturali della primaria attualizzazione di una cosa reale. È interessante, infine, annotare il fatto che tale momento veritativo di ratifica costituisce proprio la forza della realtà nell'intelle- 
zione. La verità reale essendo mera attualizzazione del reale non è un processo che ha come punto di partenza colui che apprende, bensì la cosa reale che ci possiede con la sua forza d'imposizione. Non siamo noi a possedere la verità reale, ma il contrario: «la verità reale ci ha posseduto per la forza della realtà » (Zubiri, 2008 , p. 343). Tale possesso rappresenta la struttura formale dell'intellezione, tanto che da esso dipende ogni ulteriore modo di intellezione in quanto "trascinamento" dalla verità reale.

Il giudizio affermativo, l'evidenza, di ciò che una cosa è "in realtà" conferisce all'intellezione del logos senziente il suo carattere veritativo. Ovviamente, occorrerà da subito chiarire e distinguere opportunamente il campo di tale verità rispetto a quella verità reale già introdotta e analizzata a partire dall'apprensione primordiale di realtà. Se per Zubiri il problema della verità può essere esaminato solo a partire dalle attualizzazioni nell'intellezione della realtà e, quindi, a partire dai due tipi di apprensione possibili, quella primordiale e quella duale, allora, a ogni tipo di attualizzazione corrisponderà un tipo di verità: se alla semplice attualizzazione della cosa nell'intelligenza senziente corrisponde la verità reale, allora alla ri-attualizzazione del logos corrisponde un tipo di verità distinto, che Zubiri denomina verità duale.

"Quando l'attualizzazione è immediata, la sua intellezione ha ciò che abbiamo chiamato verità reale: la ratifica formale del reale in e per se stesso. E questa verità, dicevo, è semplice. Ma quando l'attualizzazione è mediata, allora il reale rende vera [verdadea] l'affermazione non come pura e semplice realtà, ma in quanto tale realtà tra altre. Nel rendere vero [verdadear] il reale in questo modo differenziale consiste formalmente l'altro tipo di verità: la verità duale» (Zubiri, 2008, p. 723).

La verità duale consiste, dunque, nel rendere vera una cosa "tra" altre, nel ri-attualizzare in distanza la cosa, già appresa come reale. Il carattere proprio della verità duale è, pertanto, quello della coincidenzialità. Una coincidenza che non rimanda in nessun modo al significato tradizionale di adeguamento, ma al fatto che ogni intellezione del logos attualizza il "tra" della cosa reale come coincidenza di intellezione e realtà. L'intellezione del logos è, così, attualità coincidenziale. Questa non è formalmente verità, bensì ambito di verità, tanto che all'interno della stessa attualità coincidenziale oltre alla verità duale si costituisce anche l'errore.

Oltre ad avere nella coincidenzialità il suo carattere proprio, la verità duale ha anche una sua struttura complessa perché, come scrive Zubiri, «la coincidenza è il carattere di un'intellezione che "giunge" a coincidere precisamente perché "colma" la distanza tra i due termini coincidenti: tra l'intellezione affermativa, e ciò che la cosa già appresa come reale è in realtà» (Zubiri, 2008, p. 723). È questo un punto chiave di tutta l'analisi zubiriana. La struttura della verità duale è formalmente dinamica, poiché a differenza della verità reale che si ha o non si ha, alla verità duale si giunge o non si giunge in coincidenza. Tale struttura dinamica si articola in tre aspetti denominati struttura media, direzionale e formale.

Il mezzo è ciò che permette la coincidenza tra l'affermazione e la cosa ulteriormente attualizzata, e, pertanto, non può intendersi come un terzo termine che si pone in relazione tra loro, poiché sarebbe eterogeneo sia all'intellezione che alla realtà e non porterebbe alla coincidenza se non casualmente o in modo estrinseco. Allora il mezzo di coincidenza della verità duale non può che essere la dimensione campale con cui rimane attualizzata la cosa reale, rappresenta quella "in realtà" che costituisce il campo. In questo modo il mezzo non è solo qualcosa che permette la coincidenza con il reale, bensì è costitutivamente qualcosa che appartiene a tale coincidenza.

Il movimento intellettivo, tuttavia, che trascorre nel mezzo non è però da esso univocamente determinato. $\grave{E}$, infatti, un movimento che ha una precisa struttura direzionale: "andiamo "verso" quella cosa, ma "da" altre» (Zubiri, 2008, p. 735). II dinamismo dell'intellezione si compie in un percorso "da-verso" in cui i due terminali sono in qualche modo fissati: "il "verso" è ciò che la cosa che si vuole intelligire è in realtà, e il "da" sono le cose in funzione delle quali si va a intelligire affermativamente la prima» (Zubiri, 2008, p. 735). Tuttavia, la direzione dell'intellezione non è uniformemente e univocamente determinata, innanzitutto per il costituirsi stesso della direzione in quanto "sarebbe" e, in secondo luogo, per il fatto che ciò che la cosa è in realtà si presenta come esigenza: la coincidenza della direzione in quanto "sarebbe" e l'esigenza rappresenta il costituirsi nella verità duale di ciò che può chiamarsi rettitudine: "La coincidenza in quanto "coincidenza di direzione e di esigenza" ha il momento formale di rettitudine. Questo è, a mio modo di vedere, il concetto rigoroso di rettitudine" (Zubiri, 2008, p. 735). Non è difficile osservare che tale concetto di rettitudine non si identifica con la verità tout court; anzi, la verità reale non può essere in alcun modo definita come "verità retta". La rettitudine rappresenta, invece, un momento intrinseco al processo intellettivo nel quale si concre- 
tizza il carattere direzionale dell'intellezione campale, del logos, senza - peraltro - garantirne il successo. Al contrario, ciò che garantisce la rettitudine è la dinamicità strutturale dell'intellezione affermativa: il fatto cioè che l'affermazione non sia in nessun modo statica, bensì costitutivamente sia un movimento intellettivo, la cui direzionalità è polivalente.

La polivalenza direzionale costituisce un ultimo momento del movimento direzionale del "da-verso". Tale concetto di polivalenza non esprime il fatto che a partire da un unico punto di partenza vi siano o possano esserci differenti cammini conformi alla semplice apprensione per la quale si è optato, bensì esprime la polivalenza all'interno della direzionalità della verità duale. Così esemplifica Zubiri:

«Per intelligire ciò che è in realtà un uomo, posso partire dagli esseri a sé affini nella scala zoologica, ma qui si apre una pluralità di direzioni: posso andare nella direzione della fonazione, posso andare nella direzione dell'esser bipede, o posso andare nella direzione del raggruppamento; nel primo caso l'uomo sarà in realtà l'animale locuente, nella seconda direzione sarà l'animale bipede (quanto meno per eccellenza), nella terza direzione sarà l'animale sociale, ecc., ecc. In questo fascio di direzioni io mi muovo all'interno di una di esse secondo una mia opzione, ancorato certamente nella ricchezza dell'intelligito, ma in una direzione determinata soltanto da una mia opzione. Questa pluralità di direzioni non è tuttavia ciò che io chiamo polivalenza direzionale. Valenza è la qualità della coincidenza in ordine alla verità. Polivalenza consiste nel fatto che queste qualità, queste valenze, possono essere diverse all'interno di ogni direzione» (Zubiri, 2008, pp. 741-743).

Come è possibile una tale polivalenza all'interno del concetto di verità? È chiaro che qui non si tratta della verità reale, ma di quella verità duale cui si giunge in un modo o in un altro all'interno del movimento intellettivo dell'affermazione. Tale polivalenza è dovuta al fatto che l'affermazione rappresenta il ri-attualizzarsi della cosa già appresa in apprensione primordiale, e questa ri-attualizzazione non è una semplice ripetizione della prima attualizzazione, ma un vero e nuovo modo di attualità. Se l'attualità primaria consiste nella realtà pura e semplice, l'attualità nell'affermazione è un'attualità distanziata ed esigita in una determinata direzione: quest'attualità è ciò che costituisce formalmente il "sembrare":

«sembrare [in primo luogo] è un'attualità della cosa reale: è la cosa reale nella sua propria realtà che è attualizzata come sembrare. Non è sembrare realtà, ma realtà nel sembrare. E in secondo luogo, è attualità in "direzione". [...] Perché, secondo quanto abbiamo visto, "sarebbe" è formalmente direzione. [...] II sembrare, dunque, non è solo attualità direzionale ma attualità in direzione "determinata". In terzo luogo, è un'attualità della cosa reale in quanto questa esige nella sua attualità, tanto inclusivamente quanto esclusivamente, determinati "sarebbe". [...] Riunendo adesso unitariamente questi tre momenti in una sola formula, dico che sembrare è l'attualità esigenziale del reale in una direzione determinata» (Zubiri, 2008, pp. 745-747).

Ogni affermazione e, pertanto, ogni giudizio è costituito da questa attualità della cosa che è il sembrare, tanto che si può dire che il giudizio sia "l'órganon formale del sembrare» (Zubiri, 2008, p. 749). Il giudizio, tra l'altro, si attua anche nella scelta della direzione nella quale si vuole andare: l'affermazione si converte, così, da direzione in via, via di affermazione, opzione nella quale si compie un discernimento, un giudizio, un krínein. La via di affermazione è, dunque, formalmente via "optativa" e ha, in ordine alla verità, tre differenti valenze, o qualità.

La prima valenza è ciò che Zubiri denomina parità. Se in ogni affermazione vi sono da una parte la semplice apprensione e dall'altra l'attualizzazione di ciò di cui si afferma, allora è necessario che vi sia tra loro una qualche omogeneità, nella linea della coincidenza. Se non vi fosse parità, tra la direzione della semplice apprensione e le esigenze del reale non si avrebbe né coincidenza né, tanto meno, rettitudine, ma si incorrerebbe in ciò che può denominarsi "sproposito".

La valenza della parità, però, non è sufficiente da sola: occorre, secondo Zubiri, un secondo livello di qualità in ordine alla verità che dica qualcosa non solo in merito al fatto che non si incorra in uno sproposito, ma rispetto anche al suo significato. La seconda valenza è il "senso". Il senso è quella valenza della verità per la quale il movimento affermativo ricade all'interno delle possibili esigenze dell'oggetto di cui si afferma. Contrariamente la direzione cadrebbe nel vuoto, in un vuoto che avrebbe due aspetti differenti: un senza-senso e un contro-senso.

Infine vi è un terzo livello di valenza che è costituito dalla coincidenza di ciò che sembra essere e di ciò che la cosa reale è in realtà. È la dualità della verità che necessita l'attualità coincidenziale del sembrare e della realtà; anzi sembrare ed essere reale, in qualche modo, non si giustappongono tra loro ma si fondono l'uno nell'altro. La questione, però, è stabilire in che modo avviene tale attualità unitaria: vi sono, cioè, due possibili coincidenze: 
"O ciò che la cosa reale è in realtà fonda ciò che sembra esserlo, oppure ciò che sembra esserlo fonda ciò che la cosa reale è in realtà. In ambedue i casi, ripeto insistentemente perché è essenziale, vi è attualità coincidenziale. Ma la caratteristica di questa coincidenza intellettiva è nei due casi essenzialmente distinta. Nel primo caso, diciamo che l'intellezione affermativa nella sua attualità coincidenziale ha questa caratteristica che chiamiamo verità. Nel secondo caso, vi è anche attualità coincidenziale, ma la sua caratteristica è quella che chiamiamo errore. [...] La via della verità è la via secondo cui è il reale ciò che fonda il sembrare. La via dell'errore è la via secondo cui il sembrare fonda la realtà: la realtà è ciò che ci sembra» (Zubiri, 2008, p. 759).

L'attualità può, dunque, realizzarsi dando preminenza o alle esigenze del reale o subordinando a queste l'attualizzazione del sembrare, incorrendo, però, in due opposte coincidenze. Entrambi i modi sono attualizzazione e si muovono all'interno dell'ambito della verità reale, ma mentre nella via della verità il sembrare è fondato in ciò che la cosa è in realtà, nella via dell'errore si devia da questo fondamento costitutivo. Essa è sì attualità coincidenziale, ma attualità falsificata, e la falsificazione consiste nel fatto di prendere il sembrare come realtà.

Tutto il percorso che Zubiri compie all'interno della struttura dinamica del logos conduce a porre la questione di cosa sia la struttura dinamica formale della verità. La complessa dottrina della verità duale porta a stabilire che la verità non è qualcosa di staticamente determinato, ma qualcosa che "accade": «La verità accade nell'attualità intellettiva di ciò che una cosa reale è in realtà» (Zubiri, 2008, p. 771). Cosa significa accadere? Nella frase esemplificativa "questa carta è bianca" non si afferma soltanto che il bianco sta nella carta, cioè non si constata il fatto che fisicamente questa carta possiede bianchezza, ma si pone l'intellezione affermativa del fatto che questa carta è bianca, cioè l'affermazione concerne la modalità con cui si giunge all'essere della verità, come arriva ad "accadere l'attualità intellettiva della bianchezza in questa carta. Se ci si fermasse alla prima considerazione noi potremmo soltanto constatare il bianco nella carta, senza considerare la verità dell'accadere intellettivo del bianco nella stessa carta. La verità non è mai qualcosa di statico, ma «qualcosa che costitutivamente "accade"» (Zubiri, 2008, p. 771). L'accadere, così, esprime certamente il carattere dinamico di ogni attualizzazione, realizzando l'attualità del reale come sembrare, attraverso due momenti, che Zubiri deno- mina "fasi della verità". Nella frase su citata, infatti, si hanno due momenti affermativi: da una parte si afferma la realizzazione del bianco, cioè si afferma che una semplice apprensione, un "sarebbe", è ora reale; dall'altra si afferma che la realizzazione del bianco accade in questa carta, cioè si afferma che la realtà del bianco si stabilisce come reale in questa carta. Questi due aspetti dell'affermazione costituiscono due fasi della verità tra loro essenzialmente differenti.

La prima fase dell'accadere della verità consiste nel fatto di affermare la realizzazione di una semplice apprensione: "bianco" è una semplice apprensione e la sua realizzazione consiste nella sua attualizzazione indipendentemente dal fatto che lo sia in una specifica carta. Si stabilisce, così, una coincidenza del reale attuale con una semplice apprensione che Zubiri denomina autenticità. È la prima fase della verità: «l'autenticità è l'attualità coincidenziale come conformità del reale con la mia semplice apprensione» (Zubiri, 2008, p. 779). L'autenticità corrisponde, dunque, non tanto a un carattere del bianco come realtà, ma alla sua attualità intellettiva; al fatto, cioè, che corrisponda la sua attualità con la semplice apprensione, con "I' $i$ dea" che ho del bianco. Cosa comporta questa corrispondenza? Diciamo che una cosa è autentica quando nella sua attualità intellettiva realizza tutti i caratteri compresi nella semplice apprensione della cosa stes$\mathrm{sa}$, vale a dire, quando vi è una conformità di ciò che è attualizzato con la sua semplice apprensione. Nell'autenticità vi è sì la semplice apprensione, il "sembrare", ma tale sembrare è fondato nella realtà di ciò che è attualizzato: l'autenticità conforme è la coincidenza del sembrare con l'essere reale. Può, però, accadere anche il contrario: si può prendere qualcosa per come sembra esserlo, o meglio, per come sarebbe in apparenza, e questo determinerebbe il falsum dell'attualità intellettiva del reale. È falso non rispetto alla mera realtà, che in quanto tale non può mai essere né vera né falsa, ma rispetto all'intellezione del logos che stabilisce una conformità laddove vi è solo discrepanza o deformità con la semplice apprensione e, quindi, privazione di autenticità.

Passiamo, ora, alla seconda fase. È la fase della verità in cui si afferma che il predicato (il bianco) è autenticamente ciò che apprendiamo nella cosa reale (questa carta). Anche qui, dunque, la coincidenza è una conformità, ma di segno differente. Mentre, infatti, nella prima fase la conformità era tra ciò che è attualizzato con la semplice apprensione, qui la conformità si stabilisce tra la cosa reale e la sua intellezione affermativa. Nell'autenticità era il bianco che si 
misurava con la semplice apprensione del bianco, il reale nel suo sembrare si misurava con l' "idea"; qui, invece, nell'intellezione affermativa il "sembrare" cerca di misurarsi con la realtà. Una tale conformità viene denominata da Zubiri conformità di intellezione affermativa 0 , meglio, conformità di giudizio: è una conformità nella quale "quello che intelligisce I'intellezione nella sua intenzione affermativa, lo intelligisce come trovandosi realizzato nella cosa reale attualizzata» (Zubiri, 2008, p. 781). Non si tratta, dunque, né dell'affermazione di un giudizio - questa carta è bianca - né del fatto che effettivamente "questa carta è bianca", bensì si tratta di qualcosa di più: «del fatto che formalmente ed espressamente ciò che io affermo è la realizzazione stessa» (Zubiri, 2008, p. 783). La conformità di giudizio, dunque, afferma la realizzazione del predicato nella cosa di cui si giudica. È, come dice Zubiri, «una realizzazione formalmente affermata» ed è, anche, "l'affermazione di una realizzazione» (Zubiri, 2008, p. 785). La conformità di giudizio attua, così, la verità nel senso di verità del giudizio.

Una volta esaminate le due fasi nella loro diversità, occorrerà affrontare la questione della loro unità, cioè dell'unità fasica della verità duale. Secondo Zubiri, l'essenza unitaria delle due fasi è nell'essere conformità in un dinamismo che va dall'autenticità del predicato alla sua realizzazione nel soggetto già reale. $\grave{E}$ questo l'accadere della verità duale come conformità. Una conformità, pertanto, fortemente dinamica, in continuo movimento, tanto che non è possibile identificarla con il termine adeguamento. Per Zubiri ogni adeguamento è certamente conformità, ma non ogni conformità è adeguamento. L'adeguamento corrisponde a una pienezza assoluta della conformità che non si dà nella realtà. Nel dire, infatti, "questa carta è bianca" si dice qualcosa di conforme a questa carta, senza che si esprima adeguatamente il colore bianco di questa carta. L'adeguamento è, pertanto, un processo che può darsi solo in un mondo ideale, non in ciò che è "in realtà"; è un processo graduale nel quale da una parte la conformità si fa, via via, più adeguata, e dall'altra la verità nel suo movimento si dirige in modo determinato e stabilisce un approccio (enfoque) verso l'adeguamento.

«Questo - spiega Zubiri - significa non che la realtà è tale come io l'affermo, ma che anche essendolo, tuttavia, la conformità stessa è come l'indice di un cammino, la cui verità consiste nel fatto che se lo percorressimo totalmente avremmo trovato l'adeguamento che si cerca» (Zubiri, 2008, p. 801).
Ogni conformità rappresenta, così, una fase verso l'adeguamento. La natura della struttura dinamica della verità duale è, allora, una direzione verso l'adeguamento, è un indice verso ciò che accade ${ }^{4}$. L'intellezione affermativa "questa carta è bianca" costituisce una conformità che gradualmente indica un remoto adeguamento in lontananza. Di qui il fatto che ogni giudizio, ogni verità duale è strutturalmente approssimazione: un'approssimazione graduale al reale e un'approssimazione a ciò che deve essere una verità adeguata. L'intera opera del sapere umano è, per questo, un'approssimazione intellettiva alla realtà.

L'analisi di Zubiri porta con sé alcune conseguenze importanti che occorre segnalare. Innanzitutto, il fatto che ogni nostra verità concreta, così come ogni errore, è sempre parziale ci pone nella condizione di farci la domanda della parzialità rispetto a cosa. Ed è chiaro che la parzialità della verità gioca il suo ruolo rispetto all'ambito primario della verità reale. È da questo ambito, tra l'altro, che sorge e si sviluppa l'ulteriore attualità del logos. II processo intellettivo all'interno del campo di realtà ha origine da una distanza rispetto all'originaria ricchezza dell'apprensione primordiale di realtà. L'opzione per una semplice apprensione a partire da ciò che la verità reale di una cosa suggerisce è sempre una semplificazione: quando si afferma che una cosa ha un certo colore la si sta apprendendo secondo una delle tantissime possibilità di note reali della cosa stessa. Tra l'altro, la parzialità della semplice apprensione non si supera sommando a questa un'infinità di altre semplici apprensioni. L'adeguamento non può intendersi come la somma di conformità parziali, poiché esiste all'interno di ogni affermazione più realtà di quanta la stessa affermazione sia capace di affermare.

In secondo luogo, dire che la verità duale è di per sé parziale non significa relativizzare alcunché. Del resto una relativizzazione avrebbe senso all'interno di un discorso di verità assoluta, cosa che non corrisponde in alcun modo al filosofare di Zubiri: parlare di verità assoluta significherebbe poi, prima di tutto, discutere di un'intelligenza assoluta che, in quanto assoluta, sarebbe priva di $\operatorname{logos}^{5}$. Il carattere senziente dell'intellezione non è né un difetto né una perfezione: è semplicemente un fatto indiscusso sul quale si origina ogni ulteriore apprensione, compresa quella del logos che permette di attualizzare le cose "in realtà". E anche la verità reale non può essere considerata come verità in sé assoluta, a meno che non si consideri assoluto il suo carattere originario. 
Da questa posizione di parzialità, di incompletezza, di finitudine si apre l'orizzonte ultimo della verità nella ragione che si presenta, innanzitutto, come incontro. Riprendendo il De trinitate di Agostino, Zubiri afferma:

«Ricerchiamo come ricercano coloro che tuttora non hanno incontrato, e incontriamo come incontrano coloro che tuttora devono cercare, poiché quando I'uomo ha terminato qualcosa, non ha fatto altro se non cominciare» (Zubiri, 2008, p. 1235).

Perché questo recupero della nozione agostiniana? Cosa ci vuole indicare Zubiri? Innanzitutto, credo, rendere esplicita non solo la radicale limitazione della conoscenza umana, ma anche esprimere il carattere formale di tutto il conoscere che incontra la verità nel suo cammino di ricerca. "L'intellezione razionale incontra la realtà che coincide o non coincide con quell'abbozzo di possibilità» (Zubiri, 2008, p. 1230) che gli è dato dall'esperienza, e in questo incontro si compie la verità razionale, una verità appunto come incontro. Ma cosa significa incontro, qual è la specificità della verità della ragione?

La verità reale è, come si è visto nel capitolo terzo, la forma primaria di verità: è lo star presente, l'attualizzazione del reale nell'intellezione. Mentre nel logos si è dato un altro modo di verità, la verità duale, che non consisteva nell'attualizzazione del reale bensì nella sua coincidenza. Una coincidenza che nel caso della semplice apprensione portava all'autenticazione, mentre nel caso del giudizio all'affermazione del vero che Zubiri denomina veridictancia. Ora, nell'intellezione razionale non si è più di fronte a un'attualizzazione, né a una coincidenza: "Non è lo stesso intelligire ciò che qualcosa è "in realtà" e ciò che qualcosa è "nella realtà" ". L'intellezione razionale mira a conoscere in una continua ricerca, e non sarebbe conoscenza se non ricercasse incontrando sempre ciò che si è cercato in quanto principio per una ricerca ulteriore. L'incontro conduce, dunque, alla verifica di ciò che si è indagato, se esso corrisponda o meno a ciò che si cercava, tanto che si può parlare di verità razionale solo nei termini di una verificazione ${ }^{6}$.

Cosa significa verificare? La verificazione è, innanzitutto, l'incontro di ciò che si cercava, del fondamento del reale campale in un abbozzo di possibilità. In questo modo l'incontro non rappresenterebbe una semplice constatazione ma sarebbe il compimento dell'abbozzato:

«Verificare è incontrare il reale, è un compimento di ciò che abbiamo abbozzato che il reale potrebbe essere: in questo incontro e in questo compimento si fa attuale (facere) il reale nell'intellezione (verum). E in questo consiste la "veri-ficazione"» (Zubiri, 2008, p. 1276).

Il facere, dunque, della verificazione non designa tanto l'attuazione di qualcosa quanto un'attualizzazione, un'attualizzazione che si costituisce come attività intellettiva. E tuttavia, tale verificazione, lungi dall'essere un momento di staticità e di fissità dell'intelligenza, è sempre aperta, sempre dinamica: verificare è sempre un "andare verificando", e questo andare è ciò che costituisce l'esperienza, non una mera constatazione di un fatto. Questo andare verificando, questa dinamica della verifica assume, poi, un carattere preciso: è una verificazione in prova ${ }^{7}$. La prova è, come sappiamo, un modo di intellezione del reale; pertanto la prova della realtà ci dà la realtà in quanto "realtà provata". La ragione si muove all'interno di questa dinamica provando l'adeguazione della verificazione. In questo modo, la verificazione si attua soltanto nel verificare nell'esperienza quale sia il compimento dell'abbozzato, non nel verificare che la ragione addotta sia l'unica vera ragione possibile ${ }^{8}$.

Tutte le verità razionali si inseriscono, infine, in un ordine rigoroso poiché ogni forma di realtà è costitutivamente rispettiva. Ogni realtà mondana quando è molteplice rinvia nel suo stesso carattere di realtà ad altre forme di realtà: nessuna forma è la sua forma di realtà, ma lo è rispettivamente ad un'altra. Allo stesso modo, ogni verità razionale rinvia ad altre verità razionali, all'interno di un ordine che non è meramente additivo, ma formale e costitutivo, presentandosi come un sistema ${ }^{9}$.

La verità, dunque, consiste sempre per Zubiri nell'attualizzazione del reale nell'intellezione. In questo senso vi sono due forme essenziali di verità corrispondentemente alle due modalità di attualizzazione del reale: la verità reale, o semplice, e la verità duale, o coincidenziale, la quale adotta alcune forme tra cui la verificazione propria della verità razionale. La verificazione, poi, coinvolge al suo interno due aspetti essenziali, l'incontro e il compimento, che da una parte rappresentano ognuno momenti differenti della verità razionale esprimendo un proprio carattere, dall'altra la loro unità costituisce la natura intrinseca della stessa verità razionale.

La verificazione come incontro implica certamente una certa conformità con il reale, ma in un modo nuovo rispetto alla verità duale; si tratta di una conformità nel senso di "confermazione". La verità razionale, infatti, non si configura nella conformità dell'affermato con il reale - cosa che spettava appunto alla verità 
duale nel suo rapporto con il logos, con l'affermazione o il giudizio - ma consiste nella confermazione dell'affermato per il reale. Non si deve, perciò, intendere la confermazione in quanto ratificazione di un'affermazione vera, ma in quanto essa dà fermezza al reale incontrato: non è, commenta Zubiri, «la ratificazione di una verità ma la costituzione stessa della verità» (Zubiri, 2008, p. 1279). L'affermazione si trasforma in confermazione, realizzando in tal modo l'incontro come verità, come verità logica.

Ma la verificazione non è solo incontro, è anche compimento:

«come compimento la verità razionale ha un carattere differente, inseparabile dal precedente ma da esso distinto. Infatti, la verità razionale come compimento è realizzazione di possibilità» (Zubiri, 2008, p. 1289).

La verità razionale, dunque, come compimento si caratterizza in quanto attualizzazione di possibilità, e questo in un duplice significato: da una parte, infatti, l'attualizzazione si realizza per la convergenza di due possibilità, quella intellettiva e quella delle cose è, cioè, attuazione o realizzazione di un fatto; dall'altra, quando tra l'attualizzazione e la possibilità sussiste un abbozzo di possibilità, non si parla più di fatto ma di "avvenimento", in quanto attualizzazione realizzata di possibilità. Questo carattere costituisce, poi, per il filosofo spagnolo, il momento storico della verità razionale; anzi, ogni intellezione razionale come compimento, cioè in quanto realizzazione di possibilità, implica questo carattere di storicità. Ed è un carattere, precisa Zubiri, non esterno alla stessa verità. Dire che la verità razionale è storica non significa soltanto affermare che ogni verità ha storia, appartiene alla storia, bensì significa affermare un carattere che appartiene all'ordine dell'attualità: «che la verità razionale sia storica in quanto verità consiste nel fatto che l'attualizzazione stessa del reale nell'intellezione è attualizzazione compiuta» (Zubiri, 2008, p. 1291). Dunque, la storicità della verità è, innanzitutto, una modalità di attualizzazione e, come tale, appartiene all'ordine dell'intellezione.

Da un lato, dunque, la verità razionale, cioè la verificazione, ha un carattere di incontro ed è verità logica, dall'altro ha carattere di compimento ed è verità compiuta, verità storica. Dunque, questa verità è verità logica e storica. Cosa implica questa "e"? Non si tratta - chiaramente - di una simultaneità o di una successione tra verità: la "e" non ha un semplice valore copulativo. Quando Zubiri afferma che la verità razionale è logica e storica, vuole indicarne l'unità,
I'indivisibilità: la verità è all'unisono logica e storica. Ognuno dei due termini implica necessariamente e formalmente l'altro: "Vale a dire, la verità razionale è logica storicamente (compiendo) ed è storica logicamente (incontrando)»(Zubiri, 2008, p. 1295). In questo consiste l'unità intrinseca della verità razionale, un'unità che si manifesta nell'essere attualità, nel modo di dare verità (verdadear) al reale. E questa attualità, questo modo di attualizzazione altro non è che l'attività pensante. L'unità, cioè l'identità del logico e dello storico, allora, altro non è che l'essenza della stessa ragione. Una ragione senziente che conosce la realtà in quanto problema, in quanto abbozzo indagante della misura del reale nel mondo della realtà. La ragione, che si muove sempre all'interno della realtà, consiste nel misurare la realtà delle cose, nell'attualizzare la realtà come problema.

In questo modo, lungi dal potersi considerare la forma suprema di intellezione, la conoscenza razionale costituisce un modo di attualità del reale, un modo di dare verità, ed è per questo una modulazione dell'intelligenza senziente che deve la sua stessa intellezione, e quindi la sua verità, a qualcosa che la precede: all'apprensione primordiale di realtà. La conoscenza è, così, «succedanea all'intellezione dell'apprensione primordiale» (Zubiri, 2008, p. 1307), essenzialmente inferiore a tale intellezione primaria, "e questo suo carattere di succedaneo consiste precisamente e formalmente nell'essere un'attualizzazione logico-storica della realtà attualizzata come problema» (Zubiri, 2008, pp. 1307-1309).

Il compimento, così, della verità razionale è costituito da una precedenza, da un qualcosa che lo precede, dalla verità reale che è già in atto per l'apprensione primordiale. Per questo motivo il problema della ragione non è mai - come sottolinea Zubiri - quello di

«verificare se è possibile che la ragione giunga alla realtà, ma proprio il contrario: in che modo occorre mantenerci nella realtà nella quale già stiamo. Non si tratta di giungere a essere nella realtà, ma di non uscire da essa» (Zubiri, 2008, p. 1309).

Volgendo alla conclusione, sembra essere utile introdurre alcune considerazioni più teoretiche e antropologiche. La possibilità di attualizzazione di questa verificazione razionale dipende dagli incontri che all'uomo accadono durante la sua esistenza. Già da un punto di vista meramente biologico tali incontri si situano su tre generazioni attraverso le quali si dà forma concreta a quello che Zubiri chiama nelle sue lezioni su El hombre e la Verdad, il "carattere pubblico della verità"10. E 
che la verità sia pubblica è dovuto, innanzitutto, a un mero fatto biologico e naturale. Tale carattere sociale e pubblico della verità si attua attraverso una "consegna" (entrega) tra le generazioni. Ma la consegna è un modo come un altro per denominare ciò che intendiamo quando parliamo di tradizione. Ma cos'è una tradizione? Una semplice trasmissione di concetti o fatti? Certamente non si avrebbe una tradizione senza trasmissione. Ma non si può dire che la tradizione dipenda soltanto dalla semplice trasmissione. C'è forse qualcosa di più originario, di più profondo. La tradizione consiste infatti nella storicità. Una storicità intesa non come un semplice trascorrere delle cose, perché - in questo senso - non si avrebbe alcuna dimensione storica degli eventi. Vale a dire: la storicità non può mai dipendere da ciò che passa, bensì da ciò che rimane. Ciò che chiamiamo, dunque, tradizione è una presenza che permane: non ci interessa la storia per se stessa ma in quanto è pervasa da qualcosa, che è appunto ciò che chiamiamo tradizione, la quale rimanendo ci facilita nella comprensione della nostra esistenza. Ciò che succede passa, tramonta, così come tutta la realtà. E la realtà nel suo accadere storico non è ciò che costituisce la tradizione. L'esemplificazione proposta da Zubiri è, per certi versi, illuminante:

«quando il padre attua sul figlio per trasmettergli qualcosa, gli trasmette la verità. Certamente, tuttavia, l'essenziale e l'importante non è questa verità che gli trasmette, ma qualcosa di più profondo, che sono le possibilità che questa verità trasmessa apre».

Ciò che la storicità e, con essa, la tradizione favorisce è il riconoscimento di una serie di possibilità reali ed effettive che esprimono il punto di inflessione nella storia della verità.
Tale prospettiva apre, poi, a due ultime notazioni finali. La tradizione non è mai una trasmissione automatica, così come stare nella realtà non è qualcosa di spontaneo. Come ci ricorda Maria Zambrano: "A stare [nel reale] si arriva attraverso una specie di totale conquista» (Zambrano, 2007, p. 6). È una conquista che si attua nell'educazione, cioè nella libertà. Se il primo requisito della comprensione è l'accettazione della realtà così come si presenta, occorre, poi, lasciare essere ciò che si mostra senza avere la brama di impadronirsene attraverso presuntuose categorie soggettive e intellettuali, bensì mettendosi al servizio dell’oggetto, perché "[La comprensione] È una passività che costituisce l'originaria attività mia, quella del ricevere, del constatare, del riconoscere» (Giussani, 1997, pp. 140-141). Bloccare la dinamica dell'energia della ragione è anche fermare la conoscenza ${ }^{11}$. Infatti:

«Il modo con cui il reale si presenta a me è sollecitazione a qualche cosa d'altro. [...] II reale mi sollecita [...] a ricercare qualche cosa d'altro, oltre quello che immediatamente mi appare. La realtà afferra la nostra coscienza in maniera tale che questa pre-sente e percepisce qualche cosa d'altro. Di fronte al mare, alla terra e al cielo e a tutte le cose che si muovono in esso, io non sto impassibile, sono animato, mosso, commosso da quel che vedo, e questa messa in moto è per una ricerca di qualcosa d'altro" (Giussani, 1997, p. 153).

È l'attesa di infinito, di alterità, costitutiva dell'uomo che, però, non si origina mai da un'idea astratta, da una logificazione dell'intellezione, come direbbe Zubiri, ma dal reale che continuamente mi sollecita dentro di esso e fuori di esso a ricercare ciò che rimane.

\section{NOTAS}

1 Sul problema della sufficienza della conoscenza umana per la filosofia greca, cfr. Esposito, 2005, pp. 5-23.

2 Cfr. Zubiri, 1987, pp. 47-53.

3 Continua Zubiri, anticipando temi che tratterà nelle successive due parti del trittico: "Se torniamo alla cosa reale a partire dal suo concetto, vi è la verità come autenticità. Se torniamo alla cosa reale a partire da un'affermazione, vi è la verità come conformità. Se torniamo alla cosa reale a partire dalla sua ragione, vi è la verità come compimento. Come vedremo, questa terza forma non è mai stata considerata dalla filosofia classica. Autenticità, conformità e compimento sono tre forme di verità duale». (Zubiri, 2008, p. 335).

4 A questo proposito, come aveva già fatto in Naturaleza, historia, Dios, Zubiri ricorda il celebre detto di Eraclito (fr. 93) secondo cui «l'oracolo di Delfi né dichiara né occulta, ma indica, significa (semaímei), ciò che deve accadere». (Zubiri, 2008, p. 805).

5 Sull'argomento, cfr. Pintor Ramos, 1994, pp. 181-182.

6 «La verificazione è la forma propria ed esclusiva della verità dell'intellezione razionale». (Zubiri, 2008, p. 1237).

7 Cfr. Zubiri, 2008, pp. 1245-1247.

8 Una tale verifica, anzi, è impossibile per Zubiri: «una cosa è la verificazione del ragionato, un'altra la verificazione della stessa ragione. Orbene quest'ultima non è verificabile. Si può verificare la verità di cui si dà ragione, ma non si può verificare la stessa ragione che si adduce. Se si potessero verificare entrambe le cose in un solo esperimento avremmo un esperimento cruciale, un experimentum crucis. Ma questi esperimenti praticamente non esistono", (Zubiri, 2008, p. 1251).

9 Cfr. Zubiri, 2008, p. 1279.

10 Cfr. Zubiri, 1999, p. 154 e ss.

11 «Una corretta comprensione delle sfide lanciate dalla cultura contemporanea e la formulazione di risposte significative a tali sfide devono avere un approccio critico ai tentativi limitati e, in definitiva, irrazionali di restringere la sfera della ragione. II concetto di ragione deve essere invece "ampliato" per essere in grado di esplorare e comprendere» (Benedetto XVI, 2007, Discorso ai Rettori e Docenti delle Università Europee, Roma, 23/6/2007). 


\section{BIBLIOGRAFÍA}

Esposito, C., Maddalena, G., Ponzio, P. y Savini, M. (2005). Felicità e desiderio. Letture di filosofia. Bari: Edizioni di Pagina.

Giussani, L. (1997). I/ senso religioso. Milano: Rizzoli.

Pintor Ramos, A. (1994). Realidad y verdad. Salamanca: Universidad Pontificia de Salamanca.

Zambrano, M. (2007). L'atteggiamento di fronte alla realtà, Tracce di luglio/agosto 2007.
Zubiri, X. (1985) Natura, storia e Dio, Palermo: Edizioni Augustinus.

Zubiri, X. (1987). Naturaleza, historia, Dios Madrid: Alianza - Fundación Xavier Zubiri.

Zubiri, X. (1999). El hombre y la verdad. Madrid: Alianza - Fundación Xavier Zubiri.

Zubiri, X. (2002). Sobre el problema de la filosofía. En: Sobre el problema de la filosofía y otros escritos (1923-1944). Madrid: Alianza - Fundación Xavier Zubiri, pp. 17-124.
Zubiri, X. (2002). Filosofía y metafísica. En: Sobre el problema de la filosofía y otros escritos (1923-1944). Madrid: Alianza Fundación Xavier Zubiri, pp. 79-214.

Zubiri, X. (2008). Intelligenza senziente. Milano: Bompiani. 